\title{
Tune discrimination in monkeys (Cebus apella) and in rats
}

\author{
M. R. D'AMATO and DAVID P. SALMON \\ Rutgers University, New Brunswick, New Jersey
}

\begin{abstract}
Monkeys and rats were trained on an operant discrimination employing structured auditory stimuli (tunes). The rats acquired the tune discrimination very rapidly and considerably faster than the monkeys. Both species generalized the discrimination across intensity and octave transformations. Discriminative performance remained at a high level when only the first halves of the tunes were presented, but substantially less generalization occurred to the second halves. Rats trained with tones (broken or steady) required three to four times more training to reach criterion than did the rats trained with tunes. The potential of structured auditory stimuli for investigations of information-processing mechanisms in animals is pointed out.
\end{abstract}

The great preponderance of studies investigating discriminative and memorial processes in monkeys have used visual discriminative stimuli, and with good reason. It has been known for some time that, compared with similar visual discriminations, monkeys (Old and New World) have considerable difficulty acquiring discriminations based on tonal frequency or tonal patterns (see Cowey, 1968, and Wegener, 1964, for reviews). From more recent work, it appears that rhesus monkeys can acquire a simple auditory go/no-go discrimination as effectively as cats when shock avoidance serves as the source of reinforcement, but acquisition is considerably retarded if positive reinforcement (orange juice) is employed (Wegener, 1976). Acquisition of operant discriminations or discriminations based on choice behavior is also relatively poor with auditory stimuli (D'Amato, 1973), particularly when the auditory cues are not correlated with differential spatial cues (Segal \& Harrison, 1978).

The possible reasons why monkeys often find auditory discriminations so difficult within contexts in which they readily acquire visual discriminations have been enumerated in detail (Cowey, 1968; Wegener, 1964). Apart from the difference in the reinforcement modality, a number of procedural considerations have been pointed out, as well as the possibility that monkeys differ in the major ecological use to which they put auditory stimuli, that is, as cues for potential predators rather than for prey (cf. Seyfarth, Cheney, \& Marler, 1980). Monkeys might therefore be more likely to withdraw from unfamiliar

This research was supported by NIMH Grant MH-32424. We wish to thank Robin Taylor and Michael Puopolo for their valuable assistance in data collection. Correspondence may be addressed to M. R. D'Amato, Department of Psychology, Rutgers University, Busch Campus, New Brunswick, New Jersey 08903. auditory cues than approach them, in contrast to cats, for which auditory cues might more often be associated with prey.

Another consideration is that the auditory stimuli used in previous studies have been relatively simple (e.g., tone vs. white noise), even when patterns have been employed (Elliott, Frazier, \& Hayden, 1971). Possibly, such poorly structured stimuli do not make the most of the monkey's highly developed auditory cortex, which may have evolved specializations for handling temporally patterned information (cf. Beecher, Petersen, Zoloth, Moody, \& Stebbins, 1979). Unlike visual signals, a great deal of the information carried by auditory stimuli resides in their temporal patterning. By using auditory stimuli that are relatively impoverished in this regard, the monkey's capacity to discriminate among auditory stimuli may have been underestimated.

The purpose of Experiment 1 was to ascertain whether monkeys would readily acquire an operant discrimination based on structured auditory stimuli (tunes) played repeatedly. Other questions addressed were whether the tune discrimination, once learned, would generalize across intensity, octave, and other transformations.

\section{EXPERIMENT 1}

As the experiment involved a number of phases, a brief overview might be helpful. The animals were first trained on a barpress discrimination between a tune that we will refer to as "charge" ( $\mathrm{S}+$ ) and no tune. After reaching criterion, the S- tune ("gliss") was introduced and training was continued until criterion. Generalization of the discrimination across intensity transformations was then assessed. In the following phases, generalization of the discrimination was further assessed by raising $S+$ first one octave and then two, so that its range of frequencies 
was completely embedded in the frequency range of the S- tune. Finally, after reaching a preestablished criterion, generalization of the discrimination to the first and to the second halves of the $S+$ and $S$ - tunes was tested.

\section{Method}

Subjects. Two male (Pete and Roscoe) and four female monkeys (Cebus apella), ranging in age from 10 to 18 years, served as subjects in the main experiment. All had had considerable past experience with a variety of visual discriminative tasks. Dagwood, Olive, and Roscoe had had no previous laboratory auditory discrimination experience. Pete, Poe, and Spider had previously received approximately $1,300,1,500$, and 1,000 trials, respectively, on a discrete-trial go/no-go auditory discrimination task in which $\mathrm{S}+$ and $S$ - were the same cues that served as the discriminative stimuli in the present study. The level of mastery achieved in the go/no-go auditory discrimination was minimal. They were included primarily for their contribution to the generalization sections of the study (Phases 2-8). During the experiment, all animals were maintained at approximately $85 \%-95 \%$ of their full-ration body weights.

Fifi and Coco, females 17 to 18 years of age, served as subjects in the control visual-discrimination experiment. Both had had extensive past experience with a variety of two-choice visual discrimination tasks.

Apparatus. One wall of two Lehigh Valley monkey chambers (Model 1317) housed five in-line stimulus projectors, located at the four corners and midpoint of a $12-\mathrm{cm}$ square. A microswitch centered below the projector array was used to initiate a trial. Two response levers were located $23 \mathrm{~cm}$ to the left and right of the microswitch, $22 \mathrm{~cm}$ above the chamber floor. A $5 \times 3.7 \mathrm{~cm}$ display unit that could be illuminated by white light appeared above each lever. The left lever and display unit were inoperative during the present study, and the stimulus projectors were employed only in the visual discrimination control study. Noyes banana-flavored pellets $(190 \mathrm{mg})$, delivered to a food cup located on the right chamber wall, served as reinforcers. A PDP/8e computer controlled all stimulus events and data recording, except for generation of the auditory stimuli.

Two 10.2-cm, 5.5-W Jensen speakers, located $20 \mathrm{~cm}$ above each lever display unit, presented the auditory stimuli, which were produced by a Commodore PET microcomputer (Model 2001) and amplified by an audio amplifier. ${ }^{1}$ The two auditory stimuli consisted of sequences of notes that formed tunes referred to as "charge," the S+ in the present study, and "gliss," the S-. The following sequence of six frequencies comprised the charge tune: $243,324,406,481,406$, and $481 \mathrm{~Hz}$. The durations of the successive frequencies were approximately $165,165,165,330,165$, and $600 \mathrm{msec}$, respectively; approximately $45 \mathrm{msec}$ separated adjacent notes.

The "gliss" tune consisted of 17 monotonically descending and 16 monotonically ascending frequencies, the lowest and highest frequencies being approximately 718 and $8,929 \mathrm{~Hz}$, respectively. Each frequency was approximately $55 \mathrm{msec}$ in duration, with a brief, barely discernible break between successive notes. The duration of a single playing of the charge tune was $1.8 \mathrm{sec}$, and that of the gliss, 2.0 sec. Successive playings of a tune were separated by a period of time that varied randomly between .5 and $1.5 \mathrm{sec}$.

The intensity of the tunes was approximately $85 \mathrm{~dB}$ (re $.0002 \mu$ bar), as measured on the $C$ scale of a sound-level meter. (General Radio Model $1565-\mathrm{B}$ ) positioned $17 \mathrm{~cm}$ from the response panel and $20 \mathrm{~cm}$ above the floor, approximating the position of the subject's head at the time of trial initiation. The intensity of the tunes could be lowered to approximately $76 \mathrm{~dB}$ by adding a $20-\Omega$ resistor in series with the speakers. This intensity value is well above the auditory threshold of monkeys (cf. Stebbins, 1970). To reduce interference from extrancous sounds, white noise of approximately $60 \mathrm{~dB}$ was continuously present in the experimental room.

Procedure. The animals were shaped as necessary to press the right lever on a continuous reinforcement schedule. Over the next few (2-5) sessions, the animals were gradually introduced to the final format of the discriminative trials, which was as follows. A trial was initiated by completion of a fixed-ratio 10 schedule (FR 10) on the microswitch, resulting in extinction of the houselight (placing the animal in near-total darkness) and the presentation of the auditory stimulus. After two playings of the auditory stimulus, the houselight came on and the display unit above the right bar was illuminated, indicating that the bar was enabled. On S+ trials, the playing of the charge tune continued until the animal completed a fixed-interval 10-sec schedule (FI 10), which produced a reinforcement pellet and extinguished the display unit. A 30-sec intertrial interval (ITI) followed, signaled by a change in the chamber illumination. On $\mathrm{S}-$ trials, if the animal failed to press the illuminated lever for $10 \mathrm{sec}$, the trial was terminated. To discourage responding on such trials, termination of an $\mathbf{S}-$ trial was delayed for $2 \mathrm{sec}$ whenever the animal responded within $2 \mathrm{sec}$ of the scheduled termination. There were minor variations of this "punishment" procedure, tailored to the individual animal.

Responses were recorded during only the first $10 \mathrm{sec}$ of an $S+$ or an $S-$ trial. Discriminative behavior was indexed by a discrimination ratio based on the number of $S+$ responses divided by the sum of $S+$ and $S-$ responses, multiplied by 100 .

In Phase $1, \mathrm{~S}+$ consisted of the charge tune at high intensity while $S-$ was the absence of an auditory stimulus. The trial sequence was as indicated above, the only difference being that on $S-$ trials no tune was presented. It was originally planned to run all animals until they had achieved a discrimination ratio (DR) of at least $90 \%$ in Phase 1, but Dagwood and Olive failed to reach this level in a reasonable number of sessions. In this and in all subsequent phases, the daily session consisted of $24 \mathrm{~S}+$ and 24 S- trials, quasi-randomly intermixed.

During Phase $2 \mathrm{a}$, the $\mathrm{S}-$ tune, gliss, was introduced and maintained at its low intensity. Thus, in addition to other differentiating characteristics, a substantial intensity difference existed between $S+$ and $S-$. Five of the six animals were trained on the discrimination until a DR of at least $90 \%$ was reached in one session; in the case of Olive, the criterion was 2 successive days with $D R>$ $85 \%$. During Phases 1 and $2 a$, some subjects were run a few sessions (1-7) beyond the point at which they had reached criterion.

In Phase 2, the intensity of S- was increased to the level of S+, and testing was continued until the animals reached a high performance level, generally until DR $>90 \%$. In Phase $3, \mathrm{~S}+$ and Swere presented at their low intensity and all subjects were tested until a DR of at least $90 \%$ was achieved in one session.

In Phase 4, the intensity of S + and S- varied randomly between their high and low levels, which eliminated the possibility that the animals could discriminate between the two tunes solely on the basis of intensity cues. Testing was continued until a DR of at least $90 \%$ was achieved in two successive sessions. The high intensity of $S+$ and $S-$ was used in all subsequent phases of the experiment.

In Phase 5, S+ was raised one octave (the frequency of all notes doubled), and the animals were tested until a DR of $90 \%(89 \%$ in one case) was reached. In Phase 6, S+ was again raised one octave, with the result that its range of frequencies $(972-1,924 \mathrm{~Hz})$ was completely embedded in the range of frequencies of the Stune $(718-8,929 \mathrm{~Hz})$. Testing continued until the subjects reached a DR of at least $90 \%$ in one session. (Five subjects were run 1-3 sessions beyond criterion, the sixth, for 17 sessions.)

During Phases 7 and 8 , the frequency range of the $S+$ tune was as in Phase 6. Phase 7 assessed the degree to which the tune discrimination generalized to the first halves of the $S+$ and $S-$ tune. In the case of the S- tune (gliss), only the initial, descending portion of the tune was presented, the ascending portion being replaced by an equal duration of no tune. For the $S+$ tune (charge), the last two of the six notes were deleted. The subjects were tested 
with the truncated tunes until a DR of at least $90 \%$ was achieved or for a maximum of 12 sessions.

Generalization of the discrimination to the second halves of the tunes was assessed in Phase 8. In the case of S-, the animals heard only the second, ascending portion of the tune. On S+ trials, they heard only the last three of the six-note charge tune. Three of the subjects received Phase 7 first, followed by Phase 8 , and three were tested in the reverse order. After completing the first of the two phases, each subject was returned to the full tunes for three sessions, with the requirement that $\mathrm{DR}>90 \%$ on the $3 \mathrm{rd}$ day. Failing to meet the latter, the subject was continued on the full tunes until it did so.

To obtain some indication of the speed of acquisition on a comparable visual discrimination task, two subjects were run through Phases 1 and 2 using visual rather than auditory discriminative stimuli. The training procedures were the same as those described above for the auditory discrimination, with the following exceptions. In Phase 1, on S+ trials, completion of the FR 10 on the microswitch resulted in extinction of the houselight and illumination of the four outer projectors with red light, which constituted $\mathrm{S}+$. On S- trials, the projectors remained dark. Both subjects were run until they reached the criterion of two successive sessions with DR $>85 \%$.

In Phase 2, S- was the illumination of the four outer projectors with a white dot, $6 \mathrm{~mm}$ in diameter. The subjects were trained to the same criterion as that employed in Phase $2 \mathrm{a}$ of the main experiment, that is, one session with DR $>90 \%$.

\section{Results}

The salient results of the main experiment are presented in Table 1. The first column of numbers associated with each phase gives the number of sessions required to achieve criterion. The numbers in the second column are the DRs obtained during the first session of each phase. The last row of the table presents the mean (or where necessary, median) number of sessions to criterion and the mean DR.

Even though they showed little evidence of discriminating the tunes in their earlier training, the three subjects (Poe, Spider, and Pete) that entered the experiment with previous experience with the charge and gliss tunes acquired the tune vs. no tune discrimination (Phase 1) considerably faster than the other three animals. Indeed, Dagwood and Olive failed to reach the $90 \%$ criterion by the end of Phase 1, managing DRs of approximately $85 \%$ and
$89 \%$, respectively, by the end of this phase. The mean DR of the initial session of Phase $1,51.8 \%$, was close to chance expectation, indicating that the animals' barpress behavior was neither facilitated nor inhibited by the charge tune.

Two of the animals (Poe and Roscoe) showed complete generalization of the tune vs. no-tune discrimination when S- was introduced at low intensity (Phase 2a), and in spite of the small degree of generalization shown by Dagwood and Olive, the mean DR on the first session of Phase $2 a, 73.5 \%$ was significantly greater than chance expectation $[\mathrm{t}(5)=$ $2.81, \mathrm{p}<.05]$. The first column of numbers under Phase $2 a$ gives the number of sessions to reach a DR of $90 \%$, except in the case of Olive, for which the criterion was 2 days at $85 \%$ or better.

Raising the intensity of $\mathrm{S}-$ from low to high (Phase 2) did not materially depress discriminative performance, as the mean DR on the first session of this phase was $82.8 \%$. The numbers in the left column indicate the number of sessions that each animal received in Phase 2 , during which the animals achieved DRs ranging from $86 \%$ to $99 \%$.

Performance generally remained at a very high level when the low intensity of $S+$ and $S-$ was introduced (Phase 3), with half of the animals requiring only a single session to reach the $90 \%$ DR criterion. When the intensity of S+ and S- was randomly varied between their high and low values (Phase 4), performance showed some disruption but the mean DR obtained on the first transfer session $(87.5 \%)$ was extremely high. The performance criterion in this phase was two successive sessions with DR $\geqslant 90 \%$, and three animals met the criterion in the minimal number of sessions. Roscoe, whose behavior tended to be unstable, had difficulty in maintaining the $90 \%$ level for two successive sessions.

Doubling the frequency of S+ (charge) had very little effect upon the animals' discriminative behavior, as may be seen by the fact that the mean DR on the first session of Phase 5 was $88.7 \%$ and by the small number of sessions needed to reach criterion.

Table 1

Numbers of Sessions to Criterion (SC) and Discrimination Ratios (DR) on First Session for Each Phase of Experiment 1

\begin{tabular}{|c|c|c|c|c|c|c|c|c|c|c|c|c|c|c|c|c|c|c|}
\hline \multirow[b]{2}{*}{ Subject } & \multicolumn{2}{|c|}{ Phase 1} & \multicolumn{2}{|c|}{ Phase $2 \mathrm{a}$} & \multicolumn{2}{|c|}{ Phase 2} & \multicolumn{2}{|c|}{ Phase 3} & \multicolumn{2}{|c|}{ Phase 4} & \multicolumn{2}{|c|}{ Phase 5} & \multicolumn{2}{|c|}{ Phase 6} & \multicolumn{2}{|c|}{ Phase 7} & \multicolumn{2}{|c|}{ Phase 8} \\
\hline & SC & DR & $\mathrm{SC}$ & DR & $\mathrm{SC}$ & $\mathrm{DR}$ & SC & DR & SC & DR & $\mathrm{SC}$ & $\mathrm{DR}$ & $\mathrm{SC}$ & DR & SC & DR & $\mathrm{SC}$ & DR \\
\hline Poe & 12 & 49 & 1 & 91 & 3 & 86 & 1 & 99 & 2 & 97 & 1 & 92 & 5 & 61 & 2 & 77 & 3 & 58 \\
\hline Spider & 9 & 56 & 6 & 75 & 1 & 89 & 5 & 78 & 5 & 83 & 2 & 88 & 3 & 58 & 1 & 96 & 12 & 69 \\
\hline Pete & 20 & 47 & 13 & 66 & 2 & 90 & 2 & 79 & 2 & 92 & 1 & 91 & 11 & 73 & 1 & 93 & 9 & 54 \\
\hline Roscoe & 24 & 47 & 1 & 91 & 8 & 75 & 1 & 90 & 17 & 76 & 1 & 89 & 11 & 76 & 6 & 78 & $12+$ & 56 \\
\hline Dagwood & $32+$ & 53 & 48 & 64 & 1 & 86 & 2 & 83 & 7 & 82 & 1 & 91 & 7 & 71 & 1 & 94 & $12+$ & 58 \\
\hline Olive & $36+$ & 59 & 41 & 54 & 6 & 71 & 1 & 97 & 2 & 95 & 3 & 81 & 19 & 76 & $12+$ & 71 & 4 & 72 \\
\hline Average & 22.0 & 51.8 & 18.3 & 73.5 & 3.5 & 82.8 & 2.0 & 87.7 & 5.8 & 87.5 & 1.5 & 88.7 & 9.3 & 69.2 & 1.5 & 84.8 & 10.5 & 61.2 \\
\hline
\end{tabular}

Note-Poe, Spider, and Pete had had previous experience with $S+$ and $S_{-} ;$Spider, Roscoe, and Dagwood received Phase 8 before Phase 7. Phase $1=S+$ high, $S-$ no tune; Phase $2 a=S+$ high, $S-$ low; Phase $2=S+$ high, $S-$ high; Phase $3=S+l o w, S-l o w ; P h a s e ~ 4=$ random intensity; Phase 5 = charge tune raised 1 octave; Phase $6=$ charge tune raised 2 octaves; Phase $7=$ first half of tunes; Phase 8 = second half of tunes. 
When S+ was raised a second octave, however, the animals' discriminative performance was disturbed. The mean DR obtained on the first session of Phase $6,69.2 \%$, was significantly greater than chance $[t(5)=6.03, p<.01]$, but some of the animals required a substantial number of sessions to reach the criterion DR of $90 \%$ in one session, indicating that generalization of discrimination was far from complete.

Generalization to the first halves of the tunes was complete in three of the animals and somewhat less so in the others, resulting in a mean DR on the first session of Phase 7 of $84.8 \%$. Only one animal, Olive, failed to meet the $90 \%$ DR criterion within the allotted 12 sessions. Whether Phase 7 was presented before Phase 8 or afterwards seems not to have had an important effect on performance.

Considerably less generalization was obtained with the second halves of the tunes (Phase 8), although the mean DR obtained on the initial session (61.2) is significantly above chance expectation $[t(5)=3.68$, $\mathrm{p}<.02]$. Many more sessions were generally required to reach the $90 \%$ DR criterion in Phase 8 than in Phase 7, and two of the six animals failed to do so within the allotted 12 sessions.

As an indication of $\mathrm{S}+$ and $\mathrm{S}$ - response rates, each subject's mean number of responses was obtained for the first $10 \mathrm{sec}$ of $\mathrm{S}+$ and $\mathrm{S}$ - trials on the criterial or the terminal session of each phase. The group averages of these means appear in Table 2, along with the corresponding standard deviations. Although response output varied considerably among the six monkeys, there did not seem to be any consistent relationship between discriminative performance and response rate.

In the visual control experiment, Fifi and Coco reached criterion in Phase 1 in four and six sessions, respectively, and both met the criterion of Phase 2 in six sessions. Thus, the total numbers of acquisition sessions for these subjects were 10 and 12 , consid- erably below the corresponding values of the tune discrimination animals for Phases 1 and 2a.

\section{Discussion}

Although speed of acquisition of the tune discrimination, particularly in the three animals with no previous experience with $\mathrm{S}+$ and $\mathrm{S}-$, was not as rapid as one might hope, given the results of the visual discrimination study, all six monkeys did acquire the auditory discrimination. Therefore, the use of structured auditory stimuli (whose generation and manipulation have been greatly facilitated by modern microcomputers) seems to be a promising means of getting primates to acquire discriminative behavior based on auditory cues. Using such stimuli, we recently successfully trained monkeys on an auditoryvisual conditional discrimination, which we previously were unable to accomplish with simple tonal stimuli (cf. D'Amato, 1973).

We thought it was possible that repetitive exposure to the charge and gliss tunes would render them tightly organized auditory structures, just as they are for human beings, and that as a consequence the most dominant controlling aspect of the auditory cues would be their structure, rather than more simple attributes such as relative intensity, mean frequency, etc. The fact that the discriminative behavior of the animals generalized so well over intensity transformations (Phases 2-4) provides some support for this expectation in that it suggests intensity was not an important controlling feature of the tunes.

Humans readily recognize a tune after octave transformations, and the same appears to be true for the monkeys, at least in Phase 5, in which S+ was raised one octave. The discrimination was impaired when $S+$ was raised a further octave, embedding it entirely in $\mathrm{S}-$, but, interestingly enough, the experimenters themselves were occasionally confused by this transformation. A complex stimulus such as a tune possesses many controlling features, some more

Table 2

Mean Numbers of Responses on S+ and S-Trials of the Criterion or Terminal Session for Each Phase of Experiments 1 and 2

\begin{tabular}{|c|c|c|c|c|c|c|c|c|c|c|}
\hline & & Phase 1 & Phase $2 \mathrm{a}$ & Phase 2 & Phase 3 & Phase 4 & Phase 5 & Phase 6 & Phase 7 & Phase 8 \\
\hline & & \multicolumn{9}{|c|}{ Experiment 1} \\
\hline Mean & $\begin{array}{l}S+ \\
S-\end{array}$ & $\begin{array}{r}3.96 \\
.49\end{array}$ & $\begin{array}{r}4.27 \\
.50\end{array}$ & $\begin{array}{r}3.20 \\
.37\end{array}$ & $\begin{array}{r}3.26 \\
.17\end{array}$ & $\begin{array}{r}3.84 \\
.33\end{array}$ & $\begin{array}{r}3.63 \\
.26\end{array}$ & $\begin{array}{r}2.56 \\
.25\end{array}$ & $\begin{array}{r}3.39 \\
.29\end{array}$ & $\begin{array}{r}2.08 \\
.32\end{array}$ \\
\hline \multirow[t]{2}{*}{ SD } & $\begin{array}{l}\text { S+ } \\
\text { S- }\end{array}$ & $\begin{array}{r}2.08 \\
.31\end{array}$ & $\begin{array}{r}2.81 \\
.32\end{array}$ & $\begin{array}{r}1.34 \\
.17\end{array}$ & $\begin{array}{r}1.51 \\
.10\end{array}$ & $\begin{array}{r}2.65 \\
.31\end{array}$ & $\begin{array}{r}1.58 \\
.10\end{array}$ & $\begin{array}{r}1.23 \\
.16\end{array}$ & $\begin{array}{r}1.38 \\
.23\end{array}$ & $\begin{array}{l}.84 \\
.35\end{array}$ \\
\hline & & \multicolumn{9}{|c|}{ Experiment 2} \\
\hline Mean & $\begin{array}{l}\text { St } \\
\text { S- }\end{array}$ & $\begin{array}{r}4.65 \\
.80\end{array}$ & & $\begin{array}{r}6.37 \\
.46\end{array}$ & $\begin{array}{r}6.85 \\
.34\end{array}$ & $\begin{array}{r}6.37 \\
.23\end{array}$ & $\begin{array}{r}6.86 \\
.22\end{array}$ & $\begin{array}{r}6.97 \\
.39\end{array}$ & $\begin{array}{r}6.88 \\
.24\end{array}$ & $\begin{array}{r}7.14 \\
.84\end{array}$ \\
\hline SD & $\begin{array}{l}S+ \\
S-\end{array}$ & $\begin{array}{r}2.57 \\
.44\end{array}$ & & $\begin{array}{r}4.67 \\
.41\end{array}$ & $\begin{array}{r}4.99 \\
.25\end{array}$ & $\begin{array}{r}4.14 \\
.22\end{array}$ & $\begin{array}{r}4.37 \\
.25\end{array}$ & $\begin{array}{r}3.87 \\
.33\end{array}$ & $\begin{array}{r}3.91 \\
.23\end{array}$ & $\begin{array}{r}3.61 \\
.57\end{array}$ \\
\hline
\end{tabular}

Note-Phase $1=S+$ high, $S-$ no tune; Phase $2 a=S+$ high, $S-$ low; Phase $2=S+$ high, $S$ - high; Phase $3=S+$ low, $S-$ low; Phase $4=$ random intensity; Phase $5=$ charge tune raised 1 octave; Phase $6=$ charge tune raised 2 octaves; Phase $7=$ first half of tunes; Phase 8 = second half of tunes. 
compelling than others. Individuals do not always rely on the same property of the stimulus and may, according to circumstances, shift their reliance from one feature to another. In any case, the monkey's discriminative behavior, although impaired in Phase 6, was by no means destroyed, indicating that they had discriminated aspects of the tunes other than general frequency characteristics.

As might be expected from general principles of discrimination learning and the fact that humans easily recognize a tune given only its beginning (Deutsch, 1972), Phase 7 revealed strong control by the first halves of $S+$ and $S-$. A very high mean DR was obtained on the initial session of this phase, even though Olive performed atypically. The substantially poorer performance produced by the second halves of the tunes (Phase 8) also suggests that the animals were to some degree discriminating on the basis of the structure of the tunes. If the discrimination were largly based on some general differentiating characteristic of the tunes, such as tempo, one would expect more generalization to the second halves than was obtained. In a similar vein, if the animals' discriminations were based simply on the first halves of the tunes with no appreciation of the structure of the entire tune, one would expect complete generalization in Phase 7 and virtually no generalization in Phase 8. Such a pattern seems not to hold, at least not for Olive, Poe, or Spider, the latter achieving a DR of $69 \%$ on the first session of Phase 8.

\section{EXPERIMENT 2}

The present experiment was motivated by curiosity about how rats would perform on a tune discrimination closely comparable to that employed in Experiment 1 . Because of their less developed auditory cortex, one might not expect in rats a great deal of facilitation by the use of structured auditory stimuli as compared with steady tones or white noise, stimuli generally used as discriminanda in this modality. If, on the other hand, rats proved to be adept at discriminating structured auditory stimuli, this ability could prove useful in a variety of behavioral tasks. Compared with that for primates, the range of visual stimuli that can be used effectively as discriminative stimuli for rats is limited.

Essentially the same tunes were used in the present experiment as in Experiment 1, half of the animals being trained with charge as $\mathrm{S}+$ and half with gliss. In all important aspects, the procedures were comparable to those employed with the monkeys.

\section{Method}

Subjects. Eight female albino rats obtained from Blue Spruce Farms (Altamont, N.Y.) served as subjects. Their only previous experimental experience was in a $\mathrm{T}$-maze conditioned-aversion study. They were $120-150$ days of age at the start of the experi- ment, and ranged in weight from 235 to $345 \mathrm{~g}$ prior to deprivation. Throughout the experiment they were maintained at $80 \%-85 \%$ of their full-ration body weights. They were housed individually, with water constantly available.

Apparatus. Two operant chambers (Grason-Stadler, Model E3125B-100) were housed in light- and sound-attenuating enclosures. The inside of each chamber measured $20.5 \mathrm{~cm}$ in length, $20.0 \mathrm{~cm}$ in height, and $27.5 \mathrm{~cm}$ in width. Clear Plexiglas formed the side walls of the chamber, the front and rear walls were aluminum, and the floor was formed of $5-\mathrm{mm}$ bars. There were two response levers (bars) located $7.0 \mathrm{~cm}$ from the side walls and $7.5 \mathrm{~cm}$ from the grid floor, each extending $1.7 \mathrm{~cm}$ from the front wall. Located $2.5 \mathrm{~cm}$ above each bar was a translucent display unit, $2.5 \mathrm{~cm}$ in diameter, which covered a 110-V, 10-W bulb. Throughout the experiment, only the left bar and bar light were operative. Noyes food pellets ( $45 \mathrm{mg}$ ) served as reinforcers and were delivered to a food cup centered in the lower part of the front wall. A low level of illumination was provided by a 10-W bulb positioned behind the front wall of the chamber.

The auditory stimuli, essentially the same tunes as those employed in Experiment 1, were presented by a $10.2-\mathrm{cm}$ speaker located on the front wall below the left lever. With the microphone of the sound-level meter facing the left bar, $6 \mathrm{~cm}$ from the front wall and $6.5 \mathrm{~cm}$ from the grid floor, the high-intensity charge and gliss tunes measured between 81 and $84 \mathrm{~dB}$; the low-intensity tunes measured between 73 and $74 \mathrm{~dB}$. A PET microcomputer connected to each chamber produced the auditory stimuli, controlled the presentation of stimuli and trial events, and recorded the data. To reduce interference from extraneous sounds, white noise measuring about $61 \mathrm{~dB}$ within the experimental chambers was delivered from a speaker mounted in the ceiling of the test room.

Procedure. The subjects were first magazine trained and shaped to press the left bar with the light above the bar illuminated and the $\mathrm{S}+$ tune playing. The animals were then gradually introduced to the final format of a training trial as follows. After completion of a $1-\sec$ intertrial interval (ITI), the $S+$ tune was presented simultaneously with illumination of the bar light. An FI 1-sec schedule was in effect which, when completed, resulted in delivery of one 45-mg Noyes food pellet, termination of the $S+$ tune and the bar light, and entry into the ITI. Over the course of three to seven 48trial sessions, the FI schedule and the ITI were gradually increased to 10 and $30 \mathrm{sec}$, respectively, their terminal values.

Acquisition training differed from that of Experiment 1 as follows: Trials began after the 30 -sec ITI rather than being initiated by the subject. The response bar was enabled simultaneously with presentation of the auditory cue rather than being delayed until after two playings of the tune. Every response on an $\mathrm{S}-$ trial added $2 \mathrm{sec}$ to the duration of the S- trial. The performance criterion in Phase 1 was one session with DR $>85 \%$ or a maximum of five sessions. There was no Phase $2 a$; instead, in Phase 2 the $S-$ tune was introduced at its high intensity; thus, the intensity cue that was available to the monkeys in Phase 2a of Experiment 1 was not available to the rats. Finally, the acquisition criterion in Phase 2 was more stringent than in Phase $2 a$ of Experiment 1, being two successive sessions with $\mathrm{DR} \geqslant 90 \%$.

Phases 3-8, which assessed intensity and octave generalization and transfer to the first and second halves of the tunes, were conducted the same way as the corresponding phases of Experiment 1. The performance criteria were also the same, including Phase 4 , in which the criterion was two successive sessions with DR $>90 \%$.

\section{Results and Discussion}

The major results of the experiment are presented in Table 3, which follows the format of Table 1.

As with the monkeys, discriminative behavior was not significantly different from chance on the first session of Phase $1[t(7)=1.38]$. The mean DR at the end of Phase 1 was $84.5 \%$, a value close to the mean 
Table 3

Numbers of Sessions to Criterion (SC) and Discrimination Ratios (DR) on First Session for Each Phase of Experiment 2

\begin{tabular}{|c|c|c|c|c|c|c|c|c|c|c|c|c|c|c|c|c|}
\hline \multirow{2}{*}{$\begin{array}{l}\text { Sub- } \\
\text { ject }\end{array}$} & \multicolumn{2}{|c|}{ Phase 1} & \multicolumn{2}{|c|}{ Phase 2} & \multicolumn{2}{|c|}{ Phase 3} & \multicolumn{2}{|c|}{ Phase 4} & \multicolumn{2}{|c|}{ Phase 5} & \multicolumn{2}{|c|}{ Phase 6} & \multicolumn{2}{|c|}{ Phase 7} & \multicolumn{2}{|c|}{ Phase 8} \\
\hline & $\mathrm{SC}$ & DR & $\mathrm{SC}$ & DR & $\mathrm{SC}$ & DR & $\mathrm{SC}$ & DR & $\mathrm{SC}$ & DR & $\mathrm{SC}$ & DR & SC & DR & SC & DR \\
\hline 1 & 3 & 45 & 2 & 74 & 1 & 94 & 2 & 99 & 1 & 97 & 1 & 96 & 1 & 100 & 4 & 75 \\
\hline 2 & 4 & 51 & 3 & 55 & 1 & 91 & 3 & 85 & 3 & 89 & 3 & 91 & 2 & 89 & $12+$ & 57 \\
\hline 3 & 3 & 50 & 3 & 65 & 2 & 89 & 2 & 91 & 1 & 100 & 3 & 67 & 1 & 99 & $12+$ & 56 \\
\hline 4 & 5 & 70 & 4 & 50 & 1 & 97 & 2 & 92 & 1 & 97 & 1 & 90 & 1 & 98 & 3 & 70 \\
\hline 5 & 5 & 60 & 4 & 62 & 1 & 94 & 2 & 91 & 1 & 93 & 6 & 88 & 1 & 93 & 10 & 60 \\
\hline 6 & 3 & 44 & 6 & 63 & 2 & 88 & 2 & 97 & 1 & 99 & 6 & 85 & 1 & 97 & 12 & 72 \\
\hline 7 & 5 & 51 & 5 & 62 & 1 & 90 & 5 & 87 & 2 & 78 & 6 & 79 & 2 & 82 & $12+$ & 53 \\
\hline 8 & 3 & 68 & 9 & 58 & 1 & 96 & 2 & 100 & 1 & 93 & 1 & 98 & 1 & 95 & 11 & 56 \\
\hline Average & 3.9 & 54.9 & 4.5 & 61.1 & 1.3 & 92.4 & 2.5 & 92.8 & 1.4 & 93.3 & 3.4 & 86.8 & 1.3 & 94.1 & 11.5 & 62.4 \\
\hline
\end{tabular}

Note-For Subjects 4, 5, 6, and 8, S+ = gliss; Subjects 2, 5, 6, and 7 received Phase 8 before Phase 7. Phase $1=S+$ high, S- no tune; Phase $2=S+$ high, $S$ - high; Phase $3=S+$ low, $S$ - low; Phase $4=$ random intensity; Phase $5=$ charge tune raised 1 octave; Phase $6=$ charge tune raised 2 octaves; Phase $7=$ first half of tunes; Phase $8=$ second half of tunes.

DR achieved by the monkeys on the final session of Phase $1(\mathbf{8 6 . 8 \%})$, even though the monkeys had far more training (medians $=3.5 \mathrm{vs} .22 .0$ sessions).

Although disrupted by introduction of the $\mathrm{S}$ - tune in Phase 2 , the rats quickly acquired the tune discrimination. The mean number of sessions to criterion was only 4.5 , far less than the corresponding value for the monkeys in Phase 2a, even though they had had the benefit of a more lenient criterion. (To maintain comparability with the monkey data, the second criterial session is not included in the rats' results.)

The total number of sessions received in Phases 1 and 2 ranged between 5 and 12 . The slowest learner among the rats acquired the tune discrimination faster than the most rapid learner among the monkeys (Poe), which had had some 1,500 previous discrimination trials involving the identical $\mathrm{S}+$ and $\mathrm{S}-$. The uniform rapidity with which the rats learned the discrimination was unexpected.

The rats generalized virtually completely to the low intensities of S+ and S- (Phase 3), and did equally well when intensity was varied randomly (Phase 4). In both phases, $75 \%$ of the rats reached criterion in the minimum number of sessions.

Like the monkeys, the rats also generalized immediately when S+ was raised one octave (Phase 5); however, they showed substantially more generalization than the monkeys to the further octave increase in Phase $6(86.8 \%$ on the initial session vs. $69.2 \%$ for the monkeys). To evaluate this difference in generalization statistically, a difference score was formed for each rat and monkey based on the terminal session of Phase 5 and the initial session of Phase 6. The difference between the mean values $(9.1 \%$ and $23.7 \%$, respectively) was statistically significant $[\mathrm{t}(12)=2.54, \mathrm{p}<.05]$.

The pattern of results obtained in Phases 7 and 8 was very similar to that observed in Experiment 1. There was substantial generalization to the first half of the tunes, with considerably less, though significant $[t(7)=4.09 . p<.01]$, generalization to the second halves. Compared with the monkeys, the rats perhaps displayed a bit more control by the first halves of the tunes and a bit less by the second.

The mean numbers of responses executed on the first $10 \mathrm{sec}$ of $\mathrm{S}+$ and $\mathrm{S}-$ trials for the criterion or terminal session of each phase are presented in Table 2. Although the rats generally responded at a higher rate than the monkeys, like the latter there was considerable variation among the rats. The response rates of several rats fell within the range of rates observed in the monkeys.

The present experiment indicates that rats can acquire an auditory discrimination based on structured stimuli with remarkable speed, much faster than monkeys on a comparable discrimination. One obvious and possible critical difference between the rats and the monkeys is the fact that the latter subjects all had had extensive previous experience in the experimental apparatus with visual discrimination tasks, whereas the rats had had none. Would experimentally naive monkeys acquire the tune discrimination as rapidly as the rats?

We evaluated this possibility with the one experimentally naive monkey available to us. Atom, a 19month-old laboratory-born male with no previous experimental experience, was trained in Phases 1 and 2 using procedures virtually identical to those employed with the rats. At the end of the five sessions of Phase 1, with the charge tune serving as S+, Atom reached a DR of $79 \%$ (the rats had reached $84.5 \%$ ). In Phase 2, with gliss introduced at its high intensity, Atom required an additional 23 sessions to reach a DR $>90 \%$ on one session. However, his performance deteriorated thereafter, and another 20 sessions were needed before he (barely) met the criterion of two successive sessions with DR $>90 \%$. Thus, it took Atom a total of 47 sessions to acquire a level of performance that was managed in 12 sessions by the 
poorest of the eight rats. (Atom subsequently quickly learned and immediately generalized visual identity matching, indicating that, in the visual modality, he was quite a capable animal.)

The lack of previous visual discrimination experience seems, on the basis of Atom's results, not to be the sole reason why the rats acquired the tune discrimination so rapidly. Our impression is that the monkeys, unlike the rats, seemed constantly to input visual stimuli, even while the auditory discriminative stimuli were being presented, which may have interfered with their discriminative behavior. The rats' attention, on the other hand, seemed less global, more focused on the task at hand, which perhaps allowed them to profit from the variety of differentiating cues available in the tunes.

\section{EXPERIMENT 3}

In the absence of some sort of baseline acquisition data obtained with comparable procedures, the degree to which the structured auditory stimuli of Experiment 2 facilitated discriminative behavior is unclear. The present experiment addressed this issue by training rats with the procedures employed in Experiment 2, except that tones rather than tunes were used as discriminative stimuli. For one group of animals, the tones were presented continuously during a trial. For a second group, the tones were intermittent, with off times of the same durations as employed in Experiment 2 . Presumably, there would be less adaptation to the tones with the latter procedure.

\section{Method}

Subjects and Apparatus. Twelve subjects of the same description, age range, and previous experience as those employed in Experiment 2 were used.

The apparatus and equipment were the same as in Experiment 2. A tone of $363 \mathrm{~Hz}$, approximately the median frequency of the lowoctave charge tune, served as one discriminative stimulus, and a tone two octaves higher $(1,454 \mathrm{~Hz})$ served as the other. The latter tone was higher in frequency than the median frequency of the gliss tune $(1,330 \mathrm{~Hz})$.

Procedure. In the steady-tone group, the $S+$ or $S-$ tone played continuously during a trial. In the broken-tone group, the tones were presented for periods of $1.87 \mathrm{sec}$, separated by off times that, as in Experiment 2, varied randomly between .5 and $1.5 \mathrm{sec}$. The low-frequency tone was assigned as $S+$ to three animals of each group, the high-frequency tone serving this role for the other three animals. One animal in the broken-tone group was discarded in Phase 1 because of extremely low response rates that did not improve by the end of Phase 1. Except for the auditory cues, pretraining followed the same procedure as in Experiment 2. Both groups of subjects were run through Phases 1-4, using exactly the same procedures and criteria as in Experiment 2.

\section{Results and Discussion}

Table 4 presents the data for both the steady-tone and broken-tone groups. The deleterious effect of the steady tone was evident in Phase 1, none of the six animals reaching the $85 \%$ DR criterion within the
Table 4

Number of Sessions to Criterion (SC) and Discrimination Ratios (DR) on First Session for Each Phase of Experiment 3

\begin{tabular}{|c|c|c|c|c|c|c|c|c|}
\hline \multirow[b]{2}{*}{ Subject } & \multicolumn{2}{|c|}{ Phase 1} & \multicolumn{2}{|c|}{ Phase 2} & \multicolumn{2}{|c|}{ Phase 3} & \multicolumn{2}{|c|}{ Phase 4} \\
\hline & $\mathrm{SC}$ & DR & $\mathrm{SC}$ & DR & $\mathrm{SC}$ & DR & $\mathrm{SC}$ & DR \\
\hline & \multicolumn{8}{|c|}{ Steady-Tone Group } \\
\hline $\begin{array}{l}1^{*} \\
2 \\
3^{*} \\
4 \\
5 \\
6^{*}\end{array}$ & $\begin{array}{l}5 \\
5 \\
5 \\
5 \\
5 \\
5\end{array}$ & $\begin{array}{l}65 \\
69 \\
48 \\
42 \\
59 \\
53\end{array}$ & $\begin{array}{l}24 \\
20 \\
11 \\
20 \\
23 \\
13\end{array}$ & $\begin{array}{l}59 \\
44 \\
56 \\
64 \\
49 \\
50\end{array}$ & $\begin{array}{l}2 \\
2 \\
2 \\
2 \\
2 \\
1\end{array}$ & $\begin{array}{l}87 \\
85 \\
84 \\
82 \\
82 \\
92\end{array}$ & $\begin{array}{c}11 \\
6 \\
12+ \\
2 \\
2 \\
5\end{array}$ & $\begin{array}{l}80 \\
76 \\
81 \\
95 \\
91 \\
72\end{array}$ \\
\hline \multirow[t]{2}{*}{ Average } & 5.0 & 56.0 & 18.5 & 53.7 & 1.8 & 85.3 & 5.5 & 82.5 \\
\hline & \multicolumn{8}{|c|}{ Broken-Tone Group } \\
\hline $\begin{array}{l}1 \\
2 * \\
3 \\
4^{*} \\
5\end{array}$ & $\begin{array}{l}4 \\
3 \\
3 \\
3 \\
3\end{array}$ & $\begin{array}{l}54 \\
64 \\
57 \\
55 \\
62\end{array}$ & $\begin{array}{r}13 \\
7 \\
25 \\
11 \\
19\end{array}$ & $\begin{array}{l}52 \\
65 \\
51 \\
48 \\
42\end{array}$ & $\begin{array}{l}2 \\
1 \\
1 \\
1 \\
2\end{array}$ & $\begin{array}{l}84 \\
90 \\
93 \\
95 \\
88\end{array}$ & $\begin{array}{c}12+ \\
7 \\
2\end{array}$ & $\begin{array}{l}84 \\
69 \\
95\end{array}$ \\
\hline Average & 3.2 & 58.4 & 15.0 & 51.6 & 1.4 & 90.0 & 6.0 & 85.5 \\
\hline
\end{tabular}

Note-Phase $I=S+$ high, $S-$ no tone; Phase $2=S+$ high, $S-$ high; Phase $3=S+$ low, $S-$ low; Phase $4=$ random intensity. $* S+=$ high frequency.

allotted five sessions. However, the mean DR at the end of Phase 1 was $79.7 \%$, not significantly different from the $84.5 \%$ obtained in Experiment 2. As in Experiment 2, barpress behavior was not affected by the presence or absence of the tone during the first session of Phase 1. Nor was the mean DR in the first session of Phase $2(53.7 \%)$ significantly different from chance expectation. The mean number of sessions required to reach criterion in Phase 2, 18.5, was about four times that required in Phase 2 of Experiment $2[t(12)=6.78, p<.001]$.

Generalization to the low-intensity tones (Phase 3) and to the randomized intensities (Phase 4) was considerable, but in both cases substantially below the level achieved in Experiment 2. As evaluated by $t$ tests based on the difference between the initialsession DR and the DR obtained on the last session of the previous phase, significantly more generalization was obtained in Phases 3 and 4 with the tunes than with the steady tones $[\mathrm{t}(12)=2.84$ and 2.95 , respectively, ps $<.02$ ].

Acquisition was noticeably better with the broken tones, both in Phase 1 and Phase 2. Nevertheless, the mean number of sessions required to reach criterion in Phase 2 (15.0) was more than three times the number required in Experiment 2 [ $t(11)=3.58, p<.01]$. Generalization to the low-intensity tunes (Phase 3) was quite high and comparable to the level achieved in Experiment 2. Due to a programming error in Phase 4 , the data of one animal were lost. The data from the remaining four subjects suggest that generalization to random intensities was less complete with the broken tones than with the tunes of Experiment 2. 
The present results suggest that the acquisition of discriminative behavior proceeds much more slowly with simple tonal stimuli, steady or broken, than with the structured stimuli used in Experiment 2. Possibly the difference in rates of acquisition can be narrowed by employing a more divergent pair of tones than that used in the present experiment. This issue, as well as the interesting question of identifying the properties of structured auditory stimuli that serve to facilitate discrimination acquisition, must be deferred pending future research.

\section{GENERAL DISCUSSION}

The use of structured auditory stimuli (arbitrary tunes) seems promising as a means of expanding the range of discriminative stimuli available to investigators interested in information-processing mechanisms in animals. Although two of the three monkeys in Experiment 1 that had had no previous experience with auditory cues required a rather large number of sessions to learn the tune discrimination, it is likely that additional tunes would be learned much more quickly, giving the animal a repertoire of auditory discriminative stimuli that could be employed in conditional discrimination and short-term memory tasks. Moreover, it is possible that parametric adjustments would lead to substantially faster tune acquisition than was observed in these animals. It has been suggested that auditory discrimination is facilitated by placing the sound source as close as possible to the manipulandum (Segal \& Harrison, 1978). In Experiment 1 , the speaker nearest the manipulandum was located about $30 \mathrm{~cm}$ above the lever, and it is likely that acquisition would improve with a more contiguous arrangement. As a preliminary assessment of this possibility, Fifi, one of the visual-discrimination control animals of Experiment 1, was run on the tune discrimination using the same procedures and criteria as employed in Experiment 2 but with a single speaker located directly below the response lever. After 5 Phase 1 sessions, she required only 10 sessions (including the 2 criterial sessions) to reach the Phase 2 criterion. On the other hand, Coco, the other control animal of Experiment 1 trained with the speakers in their usual locations, required 21 sessions in Phase 2 to meet the same criterion.

However, contiguity of sound source and manipulandum does not guarantee that monkeys will be as sensitive to auditory cues as they are to visual cues in comparable tasks. Using a contiguous sound source and manipulandum, Thompson (Note 1) had great difficulty in training rhesus monkeys to match to sample. He concluded that the animals that finally did learn to match did not acquire a matching concept comparable to that gained through the visual modality. On the basis of this limitation and the opposite constraint demonstrated by dolphins-facile acquisition of auditory, but not visual, complex discriminations (Herman, 1980)-Thompson (Note 2) spectulated that the mammalian orders may have independently evolved analogous cognitive capacities that operate only within each species' dominant sensory modality (cf. Herman, 1980).

While this is a distinct possibility, the limitation may reside more in past experience than in structural constraints. A great deal of learning that occurs in monkeys seems to involve primarily the visual system (e.g., learning to identify edible foods, significant conspecifics, geographical environments, etc.), perhaps creating a strong set to employ visual cues in their instrumental behavior. Although experience sharpens monkeys' discriminative use of alarm calls (Seyfarth et al., 1980) and predisposes them to selectively attend to differentiating features of natural calls (Beecher et al., 1979), auditory cues may generally offer less potential in this regard. In any case, the question of whether monkeys are cognitively impoverished in the auditory modality cannot be settled until serious attempts have been made to provide them with extensive experience within that modality (cf. Dewson, 1979). This enterprise might be facilitated by the use of structured stimuli, which, if organized as integrated units, should suffer fewer restrictions due to generalization and interference than simpler auditory stimuli. By "structured" stimuli, we refer to a sequence of tones that have some degree of temporal patterning (cf. Hulse, 1978; Restle, 1970), which presumably would become organized into an integrated unit faster than a sequence of random tones.

The rapidity with which the rats of Experiment 2 learned the tune discrimination is surprising. However, being nocturnal animals, rats rely less on visual stimuli than on olfactory and auditory cues. They also seem to make extensive use of ultrasound, as infants, in mating and in aggressive behavior (Sales \& Pye, 1974). ${ }^{2}$ Their sensitivity to, and employment of, such a wide portion of the frequency spectrum might predispose rats to make efficient use of the information available in the auditory modality.

\section{REFERENCE NOTES}

1. Thompson, R. K. R. Auditory cued reversal and matchingto-sample learning by rhesus monkeys. Paper presented at the Eighth International Congress of Primatology, Florence, Italy, July 1980 .

2. Thompson, R. K. R. Nonconceptual auditory matching by a rhesus monkey reflects biological constraints on cognitive processes? Paper presented at the Northeastern Meeting of the Animal Behavior Society, Kingston, Ontario, October 1981.

\section{REFERENCES}

Beecher, M. D., Petersen, M. R., Zoloth, S. R., Moody, D. B., \& Stebrins, W. C. Perception of conspecific vocalizations by Japanese macaques. Brain, Behavior and Evolution, $1979,16,443-460$. 
Cowey, A. Discrimination. In L. Weiskrantz (Ed.), Analysis of behavioral change. New York: Harper \& Row, 1968.

D'Amato, M. R. Delayed matching and short-term memory in monkeys. In G. H. Bower (Ed.), The psychology of learning and motivation: Advances in theory and research (Vol. 7). New York: Academic Press, 1973.

Deutsch, D. Octave generalization and tune recognition. Perception \& Psychophysics, 1972, 11, 411-412.

Dewson, J. H., III. Toward an animal model of auditory cognitive function. In C. L. Ludlow \& M. E. Doran-Quine (Eds.), The neurological bases of language disorders in children: Methods and directions for research (NINCDS Monograph No. 22). Washington, D.C: Government Printing Office, 1979.

Elliotr, D. N., Frazier, L. A., \& Hayden, R. C., Jr. Relational and absolute cues in auditory discrimination by monkeys. Perception \& Psychophysics, 1971, 10, 278-282.

Herman, L. M. Cognitive characteristics of dolphins. In L. M. Herman (Ed.), Cetacean behavior: Mechanisms and functions. New York: Wiley, 1980.

Hulse, S. H. Cognitive structure and serial pattern learning by animals. In S. H. Hulse, H. Fowler, \& W. K. Honig (Eds.), Cognitive processes in animal behavior. Hillsdale, N.J: Erlbaum, 1978.

Restle, F. Theory of serial pattern learning: Structural trees. Psychological Review, 1970, 77, 481-495.

Sales, G., \& PYE, D. Ultrasonic communication by animals. London: Chapman \& Hall, 1974.

Segal, M., \& Harrison, J. M. The control of responding by auditory stimuli: Interactions between different dimensions of the stimuli. Journal of the Experimental Analysis of Behavior, $1978,30,97-106$.

Seyfarth, R. M., Cheney, D. L., \& Marler, P. Monkey responses to three different alarm cells: Evidence of predator classification and semantic communication. Science, 1980, 210, 801-803.

Stebrins, W. C. (Ed.) Animal psychophysics: The design and conduct of sensory experiments. New York: Appleton-CenturyCrofts, 1970.
Wegener, J. G. Auditory discrimination behavior of normal monkeys. Journal of Auditory Research, 1964, 4, 81-106.

WEGENER, J. G. Some variables in auditory pattern discrimination learning. Neuropsychologia, 1976, 14, 149-159.

\section{NOTES}

1. Many of the popular microcomputers incorporate soundgenerating hardware programmable in Basic. In the present application, the Basic program controlled the content and the shift frequency of a shift register, which generated a square wave output of variable frequency and fixed amplitude. The output was amplified by a conventional audio amplifier before being fed to the speakers.

2. After completion of the present research and replacement of the speakers in the rat chambers, the presence of ultrasound in the gliss and charge tunes was assessed by a Holgate ultrasound receiver. Ultrasound was detectable in the range of approximately $20-40 \mathrm{kHz}$, with an intensity peak at approximately $33 \mathrm{kHz}$. Because the ultrasound seemed to be generated by the onset of a note, it was much more noticeable with gliss than with the charge tune. The original speakers (which, unfortunately, had been discarded) were substantially inferior in quality to their replacements, and they very likely produced much less in the way of ultrasound. Moreover, because the pattern of ultrasound produced by the first and second halves of the tunes, particularly the symmetrical gliss, was very similar, had the animals been discriminating on the basis of ultrasound, substantially more generalization to the second halves of the tunes (Phase 8) should have been observed in Experiment 2. It seems unlikely, therefore, that ultrasound constituted an important discriminative component of the tune discrimination.

(Manuscript received October 20, 1981; revision accepted for publication March 3, 1982.) 